\title{
The SaeR/S Two Component System: The Security System of Staphylococcus aureus
}

\section{Owen Burroughs, Jovanka Voyich}

Burroughs, Owen and Jovanka Voyich. (2021). "The SaeR/S Two Component System: The Security System of

Staphylococcus aureus." Curiositas 1, no 1. (15 November, 2021) 4-9. DOI: 10.15788/f2021.curio1

www.montana.edu/curiositas

Made available through Montana State University's ScholarWorks

scholarworks.montana.edu 

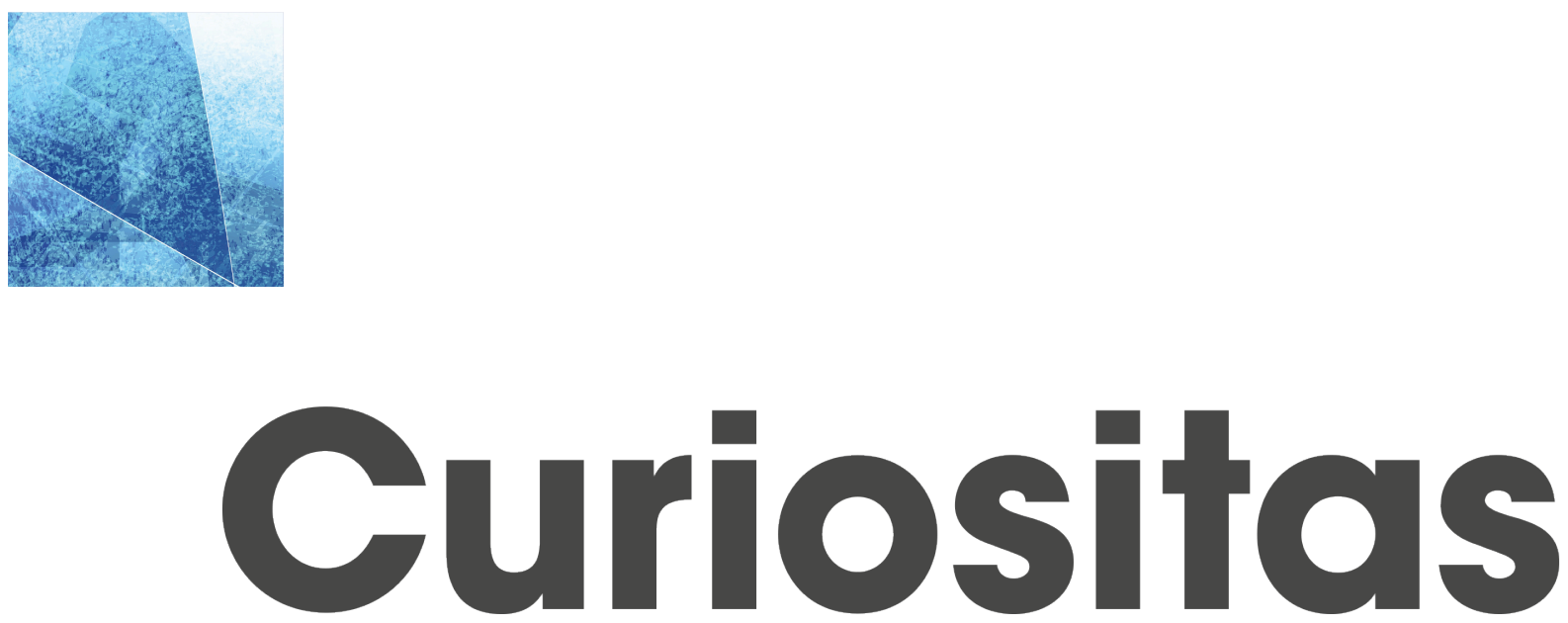

Journal of undergraduate research at Montana State University

Fall 2021

\title{
Title:
}

The SaeR/S Two Component System: The Security System of Staphylococcus aureus

\section{Authors:}

Owen Burroughs, Jovanka Voyich

\section{Author Affiliation:}

Department of Microbiology \& Cell Biology, Montana State University

\section{Volume}

Fall 2021

\section{Pages:}

\begin{abstract}
:
Staphylococcus aureus (S. aureus) is a common human pathogen that is responsible for thousands of deaths each year. The bacterium's severity is caused, in part, by its ability to detect and evade the human immune system. In this article, Owen Burroughs, an undergraduate researcher in the lab of Dr. Jovanka Voyich, describes his research into the SaeR/S two-component system, a "security system" that allows S. aureus to avoid being killed by immune cells. Over the course of Owen's research, the Voyich lab has determined that the proteins SaeP and SaeQ likely play a major role in the functioning of this security system. By helping us better understand the interactions between S. aureus and its host, this research could pave the way for new treatments and therapies for severe $S$. aureus infection.
\end{abstract}

Curiositas is an interdisciplinary research journal dedicated to presenting the breadth and depth of undergraduate research that occurs at Montana State University. The journal places a particular emphasis on showcasing overlooked domains of undergraduate research, such as the humanities and arts, alongside traditional scientific research. Curiositas is committed to the belief that research on MSU's campus does not just occur in large laboratories and research groups: it occurs in every discipline and touches every element of scholarship that occurs at MSU. Articles in Curiositas are reviewed by a faculty member in the appropriate discipline (where applicable) and by an interdisciplinary undergraduate review committee.

Montana State University Department of Microbiology \& Cell Biology 109 Lewis Hall · PO Box 173520 · Bozeman, MT 59717 406-994-2902·mcb@montana.edu
Please send questions and comments to

CuriositasJournal@montana.edu 


\title{
The SaeR/S Two-Component System: The Security System of Staphylococcus aureus
}

\author{
Owen Burroughs, Jovanka Voyich* \\ Department of Microbiology \& Cell Biology, Montana State University \\ *Faculty Sponsor
}

Staphylococcus aureus (S. aureus) is a common human pathogen that is responsible for thousands of deaths each year. The bacterium's severity is caused, in part, by its ability to detect and evade the human immune system. In this article, Owen Burroughs, an undergraduate researcher in the lab of Dr. Jovanka Voyich, describes his research into the SaeR/S two-component system, a "security system" that allows $S$. aureus to avoid being killed by immune cells. Over the course of Owen's research, the Voyich Lab has determined that the proteins SaeP and SaeQ likely play a major role in the functioning of this security system. By helping us better understand the interactions between $S$. aureus and its host, this research could pave the way for new treatments and therapies for severe $S$. aureus infection.

\section{Introduction}

Staphylococcus aureus (commonly referred to as "staph") has quickly become one of the most feared bacterial pathogens in the public consciousness. $S$. aureus infections are relatively common and typically present as mild, soft tissue infections such as impetigo. ${ }^{1}$ When allowed to progress, however, $S$. aureus causes numerous life-threatening conditions such as bacteremia (infection of the blood), meningitis, and severe pneumonia. ${ }^{1,2} S$. aureus is particularly adept at developing resistance to common antibiotics - over $95 \%$ of all strains are resistant to penicillin, for example. ${ }^{2} \mathrm{~S}$. aureus has also demonstrated that it can resist an entire class of antibiotics called beta lactams; these strains are classified as MRSA (methicillin-resistant Staphylococcus aureus). ${ }^{3}$ When MRSA was first discovered, it was almost exclusively limited to healthcare settings in patients with a compromised immune system. In the past two decades, however, hypervirulent strains of community-associated MRSA have become increasing prevalent in the United States, placing otherwise healthy individuals at risk of acquiring a potentially life-threatening infection. ${ }^{4}$ In 2017, the CDC reported nearly 120,000 MRSA cases in the United States, resulting in over 20,000 deaths. For this reason, many experts have given MRSA the notorious moniker of "superbug." 6

Researchers are in a microbial arms race with MRSA — constantly developing new treatments for MRSA infection as the bacteria becomes increasing- ly resistant. There is a clear need to develop novel treatments for severe MRSA infection. In the Voyich Lab, we study how MRSA interacts with the human immune system to help identify potential targets for antimicrobial drugs against the pathogen.

\section{S. aureus is Common but Deadly}

One of the most puzzling aspects of $S$. aureus is that it is a "commensal" bacterium, meaning it naturally colonizes a large portion of the population. Most people who are colonized with $S$. aureus will never develop an infection. ${ }^{7}$ It is estimated that $30 \%$ of Americans asymptomatically carry $S$. aureus persistently in their nose or on their skin, some of whom carry a MRSA strain. ${ }^{7}$ This leads to one of the most interesting questions in S. aureus research: why is $S$. aureus completely harmless in some cases, but deadly in others?

The answer, in part, lies in the ability of $S$. aureus to sense the human innate immune system and effectively "fight back." In most cases of S. aureus infection, the bacterium will start by attaching itself to the site of infection. Immediately afterwards, the affected tissue will release a chemical "help signal" which attracts and primes immune cells. ${ }^{9}{ }^{10}$ Typically, the first cells to respond will be neutrophils (also known as polymorphonuclear leukocytes, or PMNs) which immediately begin to engulf the bacteria in a process called "phagocytosis." ${ }^{1,4,8}$ In almost all cases, the neutrophils will effectively kill the bacteria long before it has a chance to multiply, preventing a possible infection (Figure 1). 
$S$. aureus, however, has developed numerous strategies to evade neutrophils by blocking this pathway at all stages. ${ }^{8}$ For example, $S$. aureus can: prevent chemical signals from reaching neutrophils, prevent neutrophils from being properly primed, prevent neutrophils from engulfing the bacteria, prevent neutrophils from killing the bacteria, and disrupt how neutrophils die. ${ }^{8}$ To do this, $S$. aureus produces a number of "virulence factors," which are compounds that promote an infection. ${ }^{11}$ Many of these factors are toxins that directly kill host cells. However, these toxins can signal the presence of the bacteria and attract the immune system. Therefore, in order to asymptomatically colonize its host, $S$. aureus has developed mechanisms to only produce these virulence factors when it is under threat, allowing it to remain undetected at all other times. The most significant of these mechanisms is the SaeR/S two-component system, a collection of proteins that senses the human immune system and responds with virulence factors. ${ }^{1-14}$

\section{The SaeR/S System is S. aureus's}

"Home Security System"

The SaeR/S system can be thought of as $S$. aureus's home security system. Imagine a simple security system that is comprised of three parts: a security camera, a connecting wire, and an alarm. When there is no threat present, the alarm stays silent. When the security camera sees an intruder, it transmits a signal through the connecting wire to the alarm and causes it to ring. Upon hearing the alarm, the homeowner can appropriately respond. Finally, once the threat is no longer present, the security camera uses the signaling wires to stop the alarm from ringing and the system returns to normal (Figure 2a).

The SaeR/S system contains these same three components. The protein SaeS is $S$. aureus's security camera, sensing the presence of incoming neutrophils. The protein SaeR acts as the alarm, causing $S$. aureus to produce virulence factors and respond to the neutrophils. Connecting the two proteins is the transfer of a phosphate molecule, which acts as the connecting wires (Figure 2b). Much like the security system, SaeR/S does not actively attack the neutrophils. Instead, the system detects the presence of the neutrophils and causes other molecules to mount a response.

Our lab has demonstrated that the SaeR/S system is essential for $S$. aureus to cause an infection by creating a strain of $S$. aureus that lacks SaeR/S. In the same way that a house without a security system might not detect an intruder, $S$. aureus without SaeR/S cannot detect the presence of neutrophils. This strain is more readily killed by neutro-

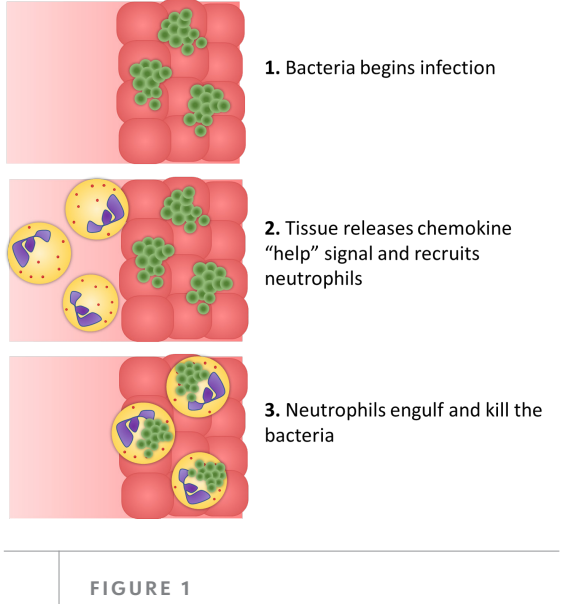

The human immune response to bacterial infection

A) A Model Home Security System

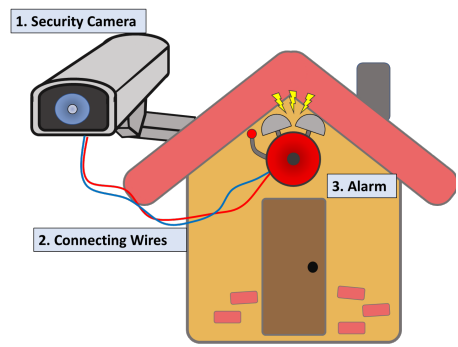

B) The SaeR/S Two-Component System

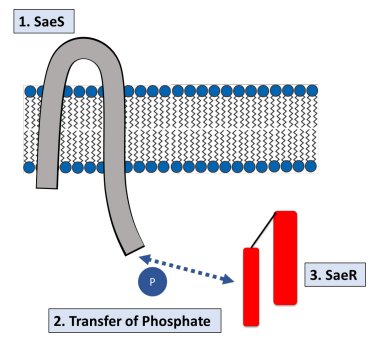

FIGURE 2

SaeR/S as S. aureus's home security system

Top: A model security system that is comprised of a security camera (1) and burglar alarm (3) connected through a series of wires (2). This is analogous to the SaeR/S two-component system (bottom) which is comprised of SaeS (1) and SaeR (3) which are connected through the transfer of a phosphate (2). In S. aureus, SaeS acts as the security camera, and SaeR acts like alarm. 


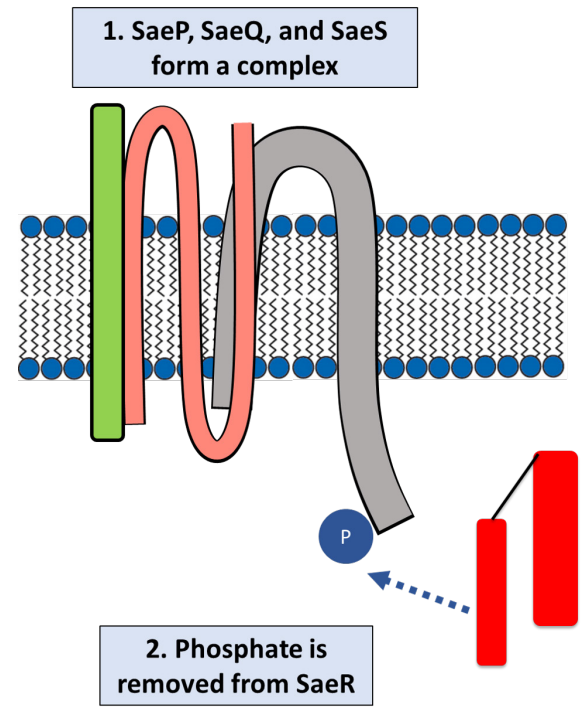

FIGURE 3

Hypothesized interaction between SaeS, SaeP, and SaeQ

Our lab hypothesized that SaeP, SaeQ, and SaeS form a complex which regulates the phosphatase activity of SaeS. Under this model, SaeR is dephosphorylated when the proteins are in the complex.

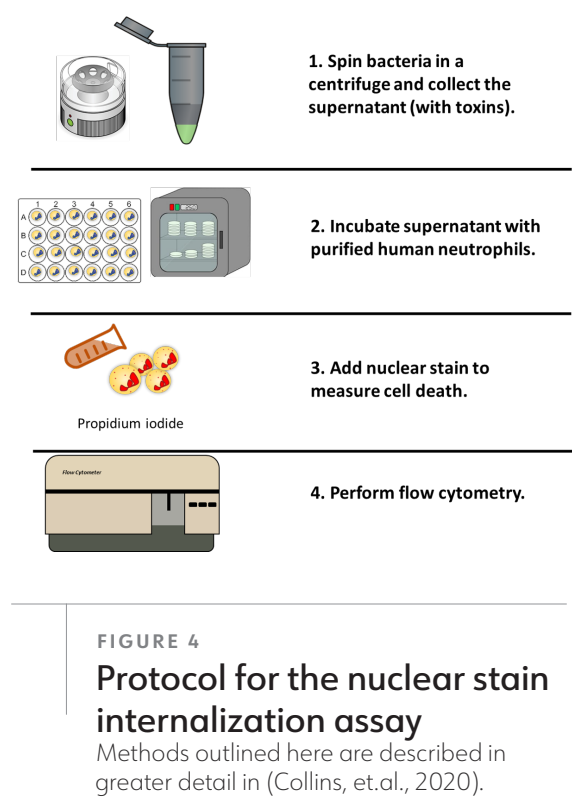

phils than unmodified S. aureus. For this reason, the SaeR/S system could be a clear target for antimicrobial drugs against $S$. aureus: inactivating this system would allow the immune system to target $S$. aureus more effectively and could decrease the severity of the infection. Our lab has investigated the transfer of phosphate from SaeS to SaeR (a process known as phosphorylation). Disrupting this process is analogous to cutting the wires between the security camera and the alarm: it would completely disable the system. Our research indicates that two auxiliary proteins (SaeP and $\mathrm{SaeQ}$ ) already modify this process of phosphorylation and could be investigated as novel therapeutic targets as an alternative to classic antimicrobials.

\section{The Auxiliary Proteins SaeP and SaeQ Modify SaeR/S}

In addition to SaeR and SaeS, the Sae system contains two additional proteins: SaeP and SaeQ. Although the Sae system has been known about for decades, these two proteins remain poorly understood. To better understand the role of each protein, we generated strains of $S$. aureus that do not contain each protein. We then ran a series of tests that compared each strain to unmodified $S$. aureus and to a strain of $S$. aureus lacking the entire SaeR/S complex.

Previous research conducted by (Jeong, et.al., 2012) indicated that the proteins SaeP and SaeQ join together and form a complex with SaeS. Once in this complex, SaeS removes the phosphate group from SaeR, preventing SaeR from continuing the production of toxins (Figure 3). Our hypothesis was that the strain of $S$. aureus lacking both SaeP and SaeQ would be more virulent than the strain with the two proteins, as it will continue producing virulence factors even after the threat subsides. In the context of the security system analogy, this would be akin to a security camera that cannot turn off its security alarm, causing the alarm to continue ringing after the threat has ceased.

Because neutrophil survival is critical for $S$. aureus to begin an infection, we tested our hypothesis with a series of experiments to measure how well each strain could survive neutrophil challenge. To begin, we purified neutrophils from freshly drawn human blood. Neutrophils in the body do not replicate on their own, and therefore cannot be grown outside of the body like many other cell types. Furthermore, neutrophils are short-lived cells, and will not survive for longer than a few hours. For both of these reasons, our lab must use a new sample of fresh blood to purify neutrophils for each experiment. Because these cells are "primary cells," meaning they were created by the human body, this method also has the added benefit of ensuring that our 
results are applicable for human infections.

\section{Featured Academic Contribution: Nuclear Stain Internalization Assays for (Collins et. al. 2020)}

This research culminated in a published paper from the Voyich Lab in the journal Frontiers in Microbiology. ${ }^{15}$ The purpose of this paper was to determine the roles of the proteins SaeP and SaeQ by testing the virulence of the mutant strains lacking each. To contribute to this paper, I conducted a series of neutrophil plasma membrane damage assays, which is a method of determining the virulence of a bacterial strain (Figure 4).

To begin this experiment, I grew three cultures of $S$. aureus: a wild-type strain (USA300), a strain lacking the proteins SaeP and SaeQ (USA300 $\Delta$ saePQ), and a strain lacking the Sae system altogether (USA300 $\triangle$ saePQRS). After growing the bacteria overnight, I spun the bacteria in a centrifuge for five minutes. As it spun, the bacteria were forced to the bottom of the tube, forming a pellet. The toxins produced by the bacteria, however, did not weigh enough to get pulled to the bottom, and so remained in the liquid at the top of the tube (known as the supernatant). By removing and filtering the supernatant, I was left with a solution that contained bacterial toxins without any bacteria.

I combined each sample of supernatant with freshly purified neutrophils and incubated for two hours. In samples without many toxins, I expected most neutrophils to survive. In samples with many virulent toxins, however, I expected a large number of the neutrophils to die.

To measure the number of surviving neutrophils, I used a technique known as nuclear stain internalization. I started by combining each sample with a dye called propidium iodide (PI), which stains the DNA within a cell. Healthy, living cells are intact and prevent the dye from reaching the DNA, but cells with disrupted membranes allow the PI to reach the DNA (Figure 5). Plasma membrane damage will eventually lead to lysis and death of the cell. Therefore, by counting the number of cells that were stained with PI, I could approximate the number of cells that were killed by the $S$. aureus toxins.

To automate this process, I used an insturment called a flow cytometer. This insturment individually counts the cells in a sample and uses a laser to determine if they have been stained with PI. Flow cytometry allowed me to measure 10,000 cells in approximately five seconds and gave me an exact percentage of cells that had been stained by PI. This percentage will be relatively low if there were not many toxins present during the incubation but will be much higher if

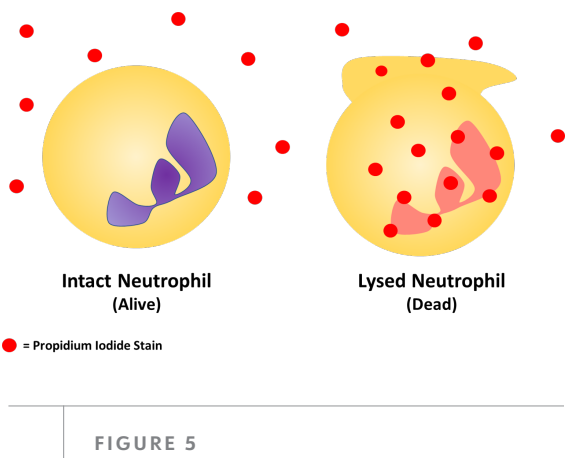

Mechanism of propidium iodide (PI) staining Intact neutrophils (left) prevent uptake of PI so the dye cannot reach the DNA. Damaged neutrophils (right) have disruptions in their membranes, allowing the dye to reach their DNA. These cells are colored red by the stain. This differentially stains a sample of neutrophils based on cell survival.

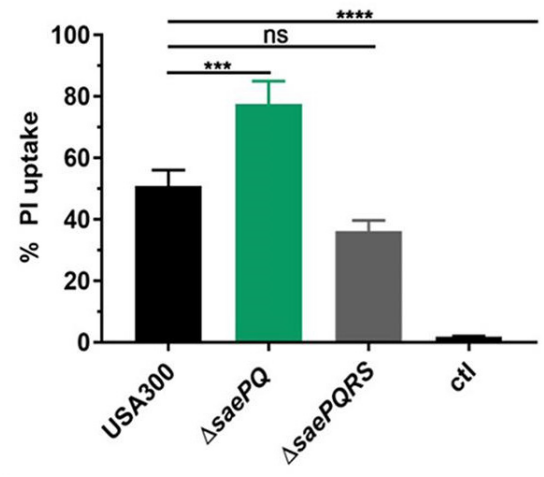

FIGURE 6

USA300 $\triangle$ sae $P Q$ demonstrates enhanced survival following neutrophil phagocytosis

Supernatant from the $\triangle$ saePQ strain causes greater PI uptake in neutrophils when compared to the wild-type USA300 strain.

Figure used from (Collins, et.al., 2020) under Creative Commons License. 


\section{Microbiology \& Cell Biology}

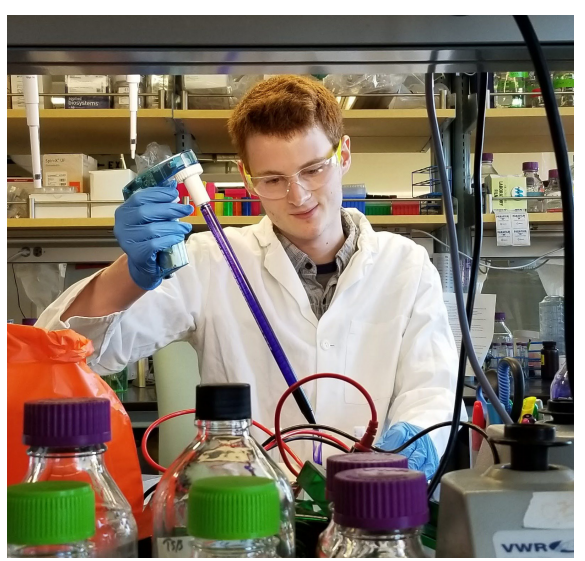

Owen Burroughs is a senior at Montana State University studying Microbiology and Political Science. Owen has worked in the Voyich Lab since 2019, studying the pathogenesis and host-pathogen interactions of Staphylococcus aureus. While at MSU, Owen has conducted multiple research projects for the INBRE and USP programs. Additionally, he spent a year working in the lab of Dr. Charlotte Odendall, a researcher at King's College London. After his undergraduate, Owen hopes to pursue an M.D/Ph.D where he can specialize in infectious diseases and public health. Originally from Bozeman, Owen loves the outdoors and enjoys skiing, hiking, and spending time with his two younger brothers. a highly virulent collection of toxins was added.

The collaborative work of myself and others generated the plot in Figure 6. Here, the bacteria lacking SaePQ (shown on the plot as $\triangle$ saePQ) caused significantly higher propidium iodide uptake than the wild-type strain (USA300). This indicates that this mutant strain produces more toxins which lyse neutrophils than the wild type. This finding supported our hypothesis that the $\triangle$ saePQ strain would be more virulent than the USA300 strain.

\section{Conclusion}

By demonstrating the importance of the proteins SaeP and SaeQ in an S. aureus infection, our research provides a potential target for new treatments against MRSA. By causing SaeP and SaeQ to form their complex with SaeS, a hypothetical new drug could significantly decrease the severity of $S$. aureus by limiting the production of toxins and virulence factors. This study underscores the importance of scientific research into host-pathogen interaction. Findings such as these will give scientists and physicians more tools to use in the fight against dangerous, antibiotic resistant pathogens such as MRSA. I find the research I do with the Voyich L ab to be incredibly rewarding, and I feel incredibly grateful to be able to conduct meaningful research as an undergraduate with such an amazing team of fellow researchers.

To read more about the conclusions of this research, please read our lab's article in Frontiers in Microbiology: DOI 10.3389/fmicb.2020.00561

\section{Acknowledgements}

Research in this publication was conducted through the Montana INBRE summer program and was supported by the National Institute of General Medical Sciences of the National Institutes of Health under award number P20GM103474 and the National Institute of Allergy and Infectious Diseases under R01-AI149491, R01A1090046, 1R56AI135039-01A1, PAR98-072, U54GM115371. 


\section{References}

1. DeLeo FR, Diep BA, Otto M. Host Defense and Pathogenesis in Staphylococcus aureus Infections. Infect Dis Clin North Am. 2009;23(1):17-34. doi:10.1016/j.idc.2008.10.003

2. Lowy FD. Staphylococcus aureus Infections. $\mathrm{N}$ Engl J Med. 1998;339(8):520-532. doi:10.1056/ NEJM199808203390806

3. Chambers HF. The changing epidemiology of staphylococcus aureus? In: Emerging Infectious Diseases. Vol 7. Centers for Disease Control and Prevention (CDC); 2001:178-182. doi:10.3201/ eid0702.010204

4. Kobayashi SD, Malachowa N, Deleo FR. Pathogenesis of Staphylococcus aureus abscesses. Am J Pathol. 2015;185(6):1518-1527. doi:10.1016/j.ajpath.2014.11.030

5. Kavanagh KT. Control of MSSA and MRSA in the United States: Protocols, policies, risk adjustment and excuses. Antimicrob Resist Infect Control. 2019;8(1):103. doi:10.1186/s13756019-0550-2

6. Ippolito G, Leone S, Lauria FN, Nicastri E, Wenzel RP. Methicillin-resistant Staphylococcus aureus: the superbug. Int J Infect Dis. 2010;14(SUPPL. 4):S7-S11. doi:10.1016/j. ijid.2010.05.003

7. Krismer B, Weidenmaier C, Zipperer A, Peschel A. The commensal lifestyle of Staphylococcus aureus and its interactions with the nasal microbiota. Nat Rev Microbiol. 2017;15(11):675-687. doi:10.1038/ nrmicro.2017.104

8. Guerra FE, Borgogna TR, Patel DM, Sward EW, Voyich JM. Epic immune battles of history: Neutrophils vs. Staphylococcus aureus. Front Cell Infect Microbiol. 2017;7(JUN):286. doi:10.3389/fcimb.2017.00286
9. Kolaczkowska E, Kubes P. Neutrophil recruitment and function in health and inflammation. Nat Rev Immunol. 2013;13(3):159-175. doi:10.1038/nri3399

10. Swain SD, Rohn TT, Quinn MT. Neutrophil priming in host defense: Role of oxidants as priming agents. Antioxidants Redox Signal. 2002;4(1):69-83. doi:10.1089/152308602753625870

11. Voyich JM, Vuong C, DeWald M, et al. The SaeR/S Gene Regulatory System Is Essential for Innate Immune Evasion by Staphylococcus aureus. J Infect Dis. 2009;199(11):1698-1706. doi:10.1086/598967

12. Geiger T, Goerke C, Mainiero M, Kraus D, Wolz C. The virulence regulator sae of Staphylococcus aureus: Promoter activities and response to phagocytosis-related signals. J Bacteriol. 2008;190(10):3419-3428.

13. Liu Q, Yeo WS, Bae T. The SaeRS twocomponent system of Staphylococcus aureus. Genes (Basel). 2016;7(10). doi:10.3390/ genes7100081

14. Nygaard TK, Pallister KB, Ruzevich P, Griffith S, Vuong C, Voyich JM. SaeR Binds a Consensus Sequence within Virulence Gene Promoters to Advance USA300 Pathogenesis. J Infect Dis. 2010;201(2):241-254. doi:10.1086/649570

15. Collins MM, Behera RK, Pallister KB, et al. The Accessory Gene saeP of the SaeR/S Two-Component Gene Regulatory System Impacts Staphylococcus aureus Virulence During Neutrophil Interaction. Front Microbiol. 2020;11(April):1-13. doi:10.3389/ fmicb.2020.00561 